\title{
Gerenciamento do cuidado de enfermagem na assistência hospitalar
}

\section{Nursing care management in hospital care}

\author{
Gyl Dayara Alves de Carvalho \\ Universidade Federal da Paraíba - UFPB - Brasil \\ gyl_dayara@hotmail.com \\ ORCID: https://orcid.org/0000-0001-6308-5142 \\ Sérgio Ribeiro dos Santos \\ Universidade Federal da Paraíba - UFPB - Brasil \\ sergio.santos2@academico.ufpb.br \\ ORCID: https://orcid.org/0000-0002-7835-3151

\begin{abstract}
Saemmy Grasiely Estrela de Albuquerque Universidade Federal da Paraíba - UFPB - Brasil saemmy.albuquerque@academico.ufpb.br ORCID: https://orcid.org/0000-0003-4944-3980
\end{abstract}
Aurilene Josefa Cartaxo de Arruda Cavalcanti
Universidade Federal da Paraíba - UFPB - Brasil aurilene_cartaxo@hotmail.com
ORCID: https://orcid.org/0000-0003-2325-4647

\author{
Recebido: 19 Janeiro 2021 \\ Revisado: 14 Junho 2021 \\ Aceito: 26 Agosto 2021
}

\section{Resumo}

Objetivo: compreender o gerenciamento do cuidado de enfermagem através do discurso de enfermeiros assistenciais. Metodologia: pesquisa qualitativa ancorada na Teoria Fundamentada nos Dados. Participaram do estudo 12 enfermeiras assistenciais de um hospital-escola de João Pessoa - PB. A análise dos dados seguiu as etapas interdependentes de codificação: aberta, axial e seletiva. Resultados: emergiu o fenômeno 'Compreendendo o gerenciamento do cuidado de enfermagem na assistência hospitalar', discutido através do modelo paradigmático de Strauss e Corbin, cujos elementos evidenciaram o gerenciamento do cuidado como um conjunto de todas as atividades desenvolvidas no cotidiano de trabalho do enfermeiro. Contribuições acadêmicas: foram trazidas novas perspectivas acerca do gerenciamento do cuidado de enfermagem que podem colaborar para seu desenvolvimento. Contribuições práticas: o estudo demonstra a relevância do papel gerencial desempenhado pelo enfermeiro e os desafios vivenciados em sua prática, suscitando a necessidade de ampliar as discussões sobre o tema e a realização de novas pesquisas em diferentes contextos, que abordem propostas para qualificação do trabalho gerencial do enfermeiro.

Palavras chave: Gestão em saúde; gerência de serviços de saúde; cuidados de enfermagem; papel do profissional de enfermagem; enfermagem.

\footnotetext{
Abstract

Purpose: to understand the management of nursing care through the speech of nursing assistants. Methodology: qualitative research anchored in Grounded Theory. Twelve nursing assistants from a teaching hospital in João Pessoa - PB, participated in the study. Data analysis followed the interdependent coding steps: open, axial and selective. Results: the phenomenon 'Understanding the management of nursing care in hospital care' emerged, discussed through the paradigmatic model of Strauss and Corbin, whose elements highlighted the management of care as a set of all activities developed in the daily work of nurses. Academic contributions: new perspectives on nursing care management have been brought up that can contribute to its development. Practical contributions: the study demonstrates the relevance of the managerial role played by nurses and the challenges experienced in their practice, raising the need to expand discussions on the theme and conduct new research in different contexts, which address proposals for qualifying managerial work the nurse.

Keywords: Health management; health services management; nursing care; role of the nursing professional; nursing.
} 


\section{Introdução}

0 processo de trabalho em saúde é descrito como um trabalho vivo em ato, que visa promover o cuidado holístico e individualizado, caracterizado por uma atividade dinâmica, imprevisível e relacional, ao qual se utiliza de diferentes tecnologias e ferramentas, pautado na ética e na ação criativa dos profissionais, no desempenho das práticas, em interação com o paciente/cliente e sua singularidade (Chagas \& Abrahão, 2017).

Logo, o cuidado integra a condição de existência, não envolvendo somente a cura, mas também os hábitos, as crenças e os valores que fazem parte da subjetividade dos sujeitos e transcendem os procedimentos técnicos e meramente curativos. Sob esse olhar, o cuidado ideal diz respeito à completa interseção entre o conhecimento técnico-científico e os aspectos individuais, interacionais, psicológicos e organizacionais, que resultam numa mistura de ciência e arte (Morais et al., 2011).

Neste sentido, o cuidado de enfermagem é visto como criativo e libertador, tendo o poder de estimular a criação e/ou regeneração da capacidade de viver de profissional e paciente, permitindo um renascimento mútuo. Cabe ao profissional de enfermagem avaliar, planejar e implementar o plano de cuidados individual, a fim de estimular as capacidades do indivíduo e facilitar a tomada de decisão (Morais et al., 2011; Cavalcante et al., 2015).

Para tanto, a literatura descreve quatro dimensões inerentes ao trabalho da enfermagem, sendo elas: o cuidado individual e com grupos em todas as fases da vida, a educação e formação profissional e educação em saúde ao usuário, o gerenciamento e organização do trabalho da enfermagem e da assistência à saúde e, por fim, a produção do conhecimento em todos os âmbitos citados anteriormente. Cada uma é indispensável para a qualificação do cuidado, foco central da profissão (Bertoncini et al., 2011). Em outras palavras, a gestão de enfermagem integra as áreas de assistência, administração, ensino e pesquisa, exigindo do profissional conhecimentos e habilidades para reconhecer a magnitude do ser humano e o processo saúde-doença, integralizando a atenção e oferecendo um cuidado holístico e resolutivo (Furuwaka \& Cunha, 2011; Chaves et al., 2012).

Ao analisar as dimensões do trabalho da enfermagem, percebe-se a existência de uma ligação entre o aspecto assistencial e o gerencial, visto que ambos são sempre solicitados conjuntamente. Assim, foi criado o conceito de gerenciamento do cuidado, que diz respeito à conexão entre o processo de cuidar e a gestão do cuidado, a fim de efetivar o atendimento às necessidades de paciente, equipe e instituição de saúde. Logo, o gerenciamento do cuidado deve ser entendido como um instrumento facilitador que efetive um modelo de produção de serviço (Santos et al., 2011; Dias et al., 2021).

As atividades de gerenciamento do cuidado são realizadas de forma sistêmica e articulada incluindo o cuidado direto e indireto, técnico e sensível, visando uma assistência integral e de boa qualidade (Christovam et al., 2012). Com isso, as atividades de gerenciamento não têm fim próprio, mas visam qualificar a assistência, através da prestação do cuidado pautado pela integralidade e pela ética, o que requer uma gerência flexível e moderna (Giordani et al., 2012).

Trazendo esses conceitos para o cuidado prestado no âmbito hospitalar, sabe-se que grande parte da demanda de assistência à saúde é realizada no nível de atenção terciária, consequentemente, engloba um contingente relevante de profissionais, em especial, de enfermeiros. Portanto, é um elemento da rede de atenção que necessita do aprimoramento do gerenciamento do cuidado, a fim de atender às novas solicitações da sociedade e da saúde como um todo, destacando ainda o papel importante dos centros formadores e alta gestão hospitalar na contribuição para implementação de estratégias de desenvolvimento de competências profissionais de seus recursos humanos (Chaves et al., 2012, Fernandes et al. 2016; Dias et al. 2021; Leal et al., 2018).

Portanto, identificou-se uma lacuna na literatura, especialmente no que diz respeito ao estabelecimento de um conceito de gerenciamento do cuidado e a caracterização de sua prática. Alguns autores reiteram essa realidade, embora existam perspectivas positivas de mudanças relacionadas à maior discussão do assunto e qualificação dos profissionais sobre o tema, é real a necessidade de estudo que embasem mudanças na realidade, em busca da sensibilização dos enfermeiros acerca da importância de articular cuidado e gerência em seu processo de trabalho (Santos et al., 2011; Santos et al., 2013; Fernandes et al. 2016; Dias et al. 2021).

Assim, a experiência prática dos autores despertou o interesse em conhecer a vivência dos enfermeiros a respeito do gerenciamento do cuidado no âmbito hospitalar, devido ao fato de observar a forte dicotomia existente acerca dessa temática, gerando a dificuldade de interligar as dimensões assistencial e gerencial em seu processo de trabalho.

Dessa forma, justifica-se a relevância do estudo, devido à presença em alguns serviços da fragmentação da assistência de enfermagem, cada vez mais emerge a necessidade de estudos que promovam discussões comprovando a ineficiência de trabalhar em uma perspectiva verticalizada, uma vez que se busca enxergar o paciente como um todo e não por partes, para fins de prestar um cuidado humanizado e de qualidade. 
Destarte, buscou-se responder o seguinte questionamento: Como o enfermeiro que atua no ambiente hospitalar compreende o gerenciamento do cuidado de Enfermagem? Logo, o estudo tem o objetivo compreender o gerenciamento do cuidado de enfermagem no ambiente hospitalar através do discurso de enfermeiros assistenciais.

\section{Revisão Teórica}

Desde que a Enfermagem foi regulamentada como profissão, suas atividades são guiadas por duas esferas: gerenciar e cuidar. A precursora Florence Nightingale foi uma das principais responsáveis por essa realidade, ao estudar e demonstrar a importância do uso da administração pelas enfermeiras para a organização e a sistematização do cuidado prestado. Apesar de dimensões pouco articuladas, elas se configuram como principais no trabalho do enfermeiro, razão por que surgiu a necessidade de serem desempenhadas conjuntamente (Christovam et al., 2012).

Prevista pela Lei do Exercício Profissional $n^{\circ}$ 7.498/86, a função gerencial é privativa do enfermeiro, englobando as ações de planejar, coordenar, executar e avaliar a assistência de enfermagem, que se configura como uma ferramenta de articulação e integração das ações e dos membros da equipe (Brasil, 1986; Montezelli et al., 2011).

0 processo de trabalho do enfermeiro no âmbito hospitalar envolve ações assistenciais, supervisão, educação em saúde e administração, que podem ser agrupadas em duas dimensões interdependentes, a assistencial e gerencial. A competência assistencial tem como objeto de intervenção as necessidades de cuidado de Enfermagem e objetiva promover a assistência integral ao paciente. Já a competência gerencial diz respeito a organização do serviço e os recursos humanos dessa categoria, para criar e empregar condições ideais de trabalho e aplicação da assistência (Montezelli et al., 2011; Massaro \& Chaves, 2009; Alves et al., 2011; Dias et al., 2021).

Destaca-se a Administração clássica como influência histórica na estruturação científica do saber em Enfermagem, demonstrada através da divisão do trabalho em classes e níveis hierárquicos, a construção de escalas, a prescrição e a distribuição de atribuições com base em normas e rotinas. Logo, torna-se necessário atentar para a gestão desse processo de trabalho, que abrange, entre outros aspectos, as relações constituídas ao longo de cada atividade. Isso porque a base do trabalho em saúde é o encontro e a partir dos impactos advindos dele, produzem-se respostas positivas ou negativas para os problemas de saúde apresentados (Alves et al., 2011; Merhy \& Chakkour, 1997).

Sabe-se que, o modo como o enfermeiro gerencia sofre interferência de aspectos históricos, sociais, políticos e tecnológicos e das características da clientela atendida. Além disso, as dificuldades que os enfermeiros enfrentam para gerenciar são reflexos das divergências construídas no cotidiano de trabalho, tendo em vista a distância entre o que aprendem durante a formação acadêmica e o que as instituições requerem deles. Por essa razão, o planejamento da assistência de Enfermagem é a base fundamental para se efetivar esse processo (Montezelli et al., 2011; Massaro \& Chaves, 2009).

0 enfermeiro desenvolve ações diretas e indiretas com os pacientes e se envolve em todas as decisões relacionadas ao cuidado dispensado ao paciente e família. Nesse contexto, é importante destacar o trabalho em equipe como primordial para a qualidade da terapêutica instituída, porém em alguns cenários ainda percebe-se a valorização das ações gerenciais em prejuízo das práticas assistências de cuidado direto revelando a falta da dialética entre os processos de cuidar e gerenciar (Pires, 2009; Fernandes et al., 2016).

Vale salientar que, o fazer e o aprender são indissociáveis no trabalho em saúde, de modo que os trabalhadores são também produtores de conhecimento, o que requer, além de uma gestão participativa, a construção de espaços interdisciplinares de estudo e pesquisa para a construção de saberes, valores e para a avaliação constante da assistência prestada e das formas de promover saúde, visto que o cuidado integral engloba muito mais do que procedimentos, pois envolve a defesa da vida e do bem-estar do indivíduo (Faria \& Araujo, 2010; Brasil, 2013).

Para gerenciar o cuidado efetivamente, o enfermeiro deve planejar a assistência em sua unidade de trabalho. Essa é uma ação contínua, que reflete na realização de uma assistência qualificada e melhora a utilização tanto dos recursos financeiros quanto dos humanos, além de contribuir com o relacionamento entre gestores, usuários e organização (Kurcgant, 2016; Marquis \& Huston, 2015). Esse planejamento se concretiza por meio da Sistematização da Assistência de Enfermagem (SAE), que requer que o enfermeiro oriente e treine a equipe de Enfermagem e conheça a individualidade de cada paciente, a fim de oferecer um cuidado holístico e direcionado (Chaves et al., 2012).

Outro elemento essencial no processo de gerenciamento é a comunicação adequada, que interfere na eficiência e na eficácia da realização de tarefas, bem como na motivação e satisfação dos profissionais, além da troca adequada das informações por meio da interação interpessoal e construção de vínculos com a equipe de enfermagem (Santos \& Bernardes, 2010; Santos et al., 2012; Howard et al, 2018). 
A liderança também é uma ferramenta importante no trabalho do enfermeiro, já que influencia o gerenciamento das atividades, o relacionamento e o envolvimento da equipe e a qualidade do cuidado prestado. Destaca-se a necessidade crescente de aproximação de instituições de ensino superior e as organizações de saúde, a fim de alinhar o perfil de profissional desejável para atuar com mais autonomia e protagonismo onde estiver inserido (Amestoy et al., 2010; Montezeli et al., 2016). Destarte, o gerenciamento visa a qualificação do cuidado, porém a articulação entre as dimensões do cuidado nem sempre acontece facilmente, tendo em vista a compartimentalização das demandas e das ações no cotidiano do trabalho da Enfermagem (Haussmann \& Peduzzi, 2009; Fernandes et al., 2016; Dias et al., 2021).

Um estudo identificou oito atividades realizadas no cotidiano de trabalho do enfermeiro, no âmbito da gerência do cuidado: dimensionamento da equipe de Enfermagem, liderança no setor de trabalho, planejamento da assistência de Enfermagem, atividades de ensino para a equipe, gerenciamento de recursos materiais, coordenação do cuidado, realização de procedimentos mais complexos e avaliação final das ações realizadas (Santos et al., 2013).

Outros estudos acrescentam ainda: a elaboração de escala, o remanejamento de funcionários, a busca de leitos disponíveis em outros setores ou hospitais, a realização da gestão da equipe de Enfermagem e avaliação de desempenho da mesma, coordenação e articulação das ações dos diversos profissionais nas unidades de internação, bem como a integração de todas as dimensões - assistência, gerência, ensino e pesquisa - o que requer não só conhecimentos voltados para o gerenciamento do cuidado, mas também a articulação com toda a instituição, com vistas a contribuir com o seu sucesso (Santos et al., 2011; Montezelli et al., 2011; Furukawa \& Cunha, 2011; Haussmann \& Peduzzi, 2009; Fernandes et al., 2016).

Uma análise crítico-reflexiva acerca da separação entre as ações de cuidado e o gerenciamento em Enfermagem, revelou que essa divisão advém do processo formativo, que historicamente, enfatiza o fortalecimento de habilidades técnicas e destreza manual, associadas a separação das atividades teóricas das práticas nos cursos de graduação, deixando em segundo plano o conhecimento psicossocial do ser humano. Esses aspectos resultaram em dificuldades para a prática do enfermeiro, tendo em vista que o saber gerencial passou a ser incluído e necessário ao seu trabalho e requer habilidades individuais, relacionais e organizacionais para atender efetivamente as demandas do cuidado (Garlet et al., 2006).

Ressalta-se a importância de atualização dos conteúdos ensinados durante a formação acadêmica do enfermeiro, com maior ênfase para as abordagens inovadoras no âmbito do gerenciamento do cuidado, a promoção e educação em saúde, ao trabalho em equipe, liderança e as aptidões requeridas pelos novos espaços de atuação profissional, distanciando-se da visão meramente assistencialista, com rotinas rígidas e prestação do cuidado centrado na doença, tanto no âmbito hospitalar como da Atenção Primária a Saúde (Santos et al., 2013; Metelski et al., 2020; Soder et al., 2020).

Ao romper essa dicotomia criam-se novas possibilidades de administrar e, consequentemente, de oferecer uma assistência bem qualificada. Para isso, é necessário esclarecer os conflitos existentes no ser humano e a função de gerência, com vistas à reflexão e à realização de um trabalho administrativo voltado para o elemento central, que é o cuidado (Montezelli et al., 2011; Fernandes et al., 2016).

0 ideal seria encontrar um ponto de equilíbrio no desempenho dessas funções, de modo a desenvolver um trabalho gerencial eficaz e democrático e dispensar um cuidado ao indivíduo de forma humanizada e pautada pela integralidade. Essa junção apresenta-se, portanto, como um paradigma da Enfermagem que vem sendo construído e denomina-se gerenciamento do cuidado (Christovam et al., 2012).

\section{Metodologia}

Trata-se de um estudo qualitativo, ancorado no referencial teórico-metodológico da Teoria Fundamentada nos Dados (TFD), realizado em um hospital público federal da rede EBSERH, localizado no município de João Pessoa - PB, Brasil, o qual foi escolhido por ser um hospital geral e pela viabilidade de acesso pelas pesquisadoras.

Os participantes do estudo foram enfermeiros que atuavam no referido hospital, escolhidos como público-alvo da investigação tendo em vista serem os líderes da equipe de enfermagem. Procurou-se abranger os enfermeiros de todas as unidades de internação, a saber: clínica médica, clínica de doenças infectocontagiosas, clínica pediátrica, unidade de terapia intensiva e clínica obstétrica. Realizou-se uma abordagem durante o horário de trabalho, quando esses profissionais foram inteirados sobre o objetivo da pesquisa e convidados a participar da investigação. Após o aceite do convite, cada entrevista foi agendada previamente conforme disponibilidade do participante. 
O quantitativo de participantes levou em consideração o critério de saturação teórica, o qual, na teoria fundamentada, consiste no momento em que o material obtido consegue responder às questões de investigação, não mais ocasionando divergências significativas ao que se encontra consolidado (Strauss \& Corbin, 2008). Após a décima segunda entrevista percebeu-se que havia sido atingido este critério e que as falas começavam a repetir os achados obtidos.

Os dados foram coletados por meio da técnica de entrevista semiestruturada, que foram gravadas e em seguida transcritas e consolidadas em um documento digitalizado, tendo duração média de 30 minutos. As entrevistas partiram do seguinte questionamento: Para você, o que significa Gerenciamento do Cuidado?

0 estudo foi realizado com doze enfermeiros assistenciais, todos eram do sexo feminino, o que demonstra que o serviço de Enfermagem continua arraigado por suas influências históricas, no que diz respeito ao gênero e à prevalência feminina (COFEN, 2017). As idades variaram entre 31 e 57 anos e seu tempo de formação variou de oito a 32 anos. 0 tempo de trabalho no ambiente hospitalar foi de três meses a 32 anos. No que diz respeito à qualificação, todas afirmaram ter cursado pós-graduação, destas, oito profissionais eram especialistas e quatro eram mestres, com o predomínio da área de interesse em saúde coletiva e saúde da família. Merece destaque também a busca pela qualificação da Enfermagem, visto que todos os entrevistados eram pós-graduados, o que é algo bastante positivo em relação ao desenvolvimento da profissão. Salienta-se a diversidade na caracterização dos participantes, tanto em relação à idade, quanto ao tempo de formação e de experiência no âmbito hospitalar.

Em relação a realização de algum curso ou capacitação sobre administração ou gerenciamento em Enfermagem, nove enfermeiras afirmaram que nunca haviam tido essa experiência e apenas três disseram ter realizado algum tipo de aprofundamento sobre o assunto. Apesar de ser uma temática relevante e presente na prática profissional, esse dado denota sua desvalorização, algo negativo tanto por parte do interesse profissional quanto da própria instituição de saúde e a busca pela qualificação de serviços prestados.

A análise do material empírico proveniente das entrevistas foi baseada na TFD nos Dados, a qual tem como base a descrição dos fenômenos em estudo para a interpretação de dados mais abstratos e desenvolvimento de uma teoria. Este processo ocorre por meio de três etapas interdependentes: codificação aberta, codificação axial e codificação seletiva. Esse processo objetiva reduzir os dados e facilitar a visualização da teoria (Strauss \& Corbin, 2008; Lewis-Pierre et al., 2017).

A codificação aberta ocorreu através de leituras do material e sua codificação, destacando palavras ou expressões que transmitissem a essência dos discursos. Na etapa de codificação axial, os códigos preliminares foram reordenados, em um nível maior de abstração, a fim de formar as categorias (Strauss \& Corbin, 2008).

Depois de constituídas as categorias, elas passaram pelo processo de comparação, conexão e definição do modelo paradigmático, o qual se caracteriza pela identificação de seis elementos: fenômeno ou ideia central, condições causais, contexto, condições intervenientes, estratégias de ação/ interação e as consequências de todos esses eventos (Strauss \& Corbin, 2008; Dantas; Leite; Lima; Stipp, 2009). Na terceira etapa de codificação seletiva, foram estabelecidas junções entre categorias, sistematização, análise e abstração, para o reconhecimento da categoria central (Strauss \& Corbin, 2008).

Esse processo de obtenção do material empírico foi lento e pautado em intensa reflexão, de modo que os códigos foram sendo identificados, nomeados e agrupados até se chegar às categorias ou fenômenos e aos elementos do modelo paradigmático que deram vida a cada um deles.

Quanto aos princípios éticos, este estudo seguiu rigorosamente a Resolução $n^{\circ}$ 466/2012, do Conselho Nacional de Saúde, que aborda a pesquisa que envolve seres humanos (Brasil, 2012). 0 projeto foi submetido ao Comitê de Ética e Pesquisa e obteve aprovação sob Certificado de Apresentação para Apreciação Ética.

\section{Resultados}

Os enfermeiros participantes do estudo assinaram o termo de consentimento livre e esclarecido e receberam uma cópia do mesmo. As entrevistas foram identificadas através de códigos numéricos, por exemplo: Enf. 1, Enf. 2, e assim sucessivamente, a fim de garantir o sigilo das informações.

Após a análise dos dados, o fenômeno que emergiu foi: Compreendendo o gerenciamento do cuidado de Enfermagem, o qual está descrito a seguir com a aplicação do modelo paradigmático de Strauss e Corbin (2008) e seus elementos, ancorado nos discursos dos profissionais participantes do estudo e na literatura pertinente. A Figura 1 apresenta o diagrama representativo dos seis elementos do modelo paradigmático, revelando as condições causais, contexto, condições intervenientes, estratégias e consequências, em que se evidencia a interligação dos componentes periféricos ao 
modelo paradigmático, uma vez que as ideias expressas nos anéis externos contribuem para a ideia central.

Figura 1 - Diagrama representativo do modelo paradigmático de Strauss e Corbin (2008) para o Fenômeno: Compreendendo o gerenciamento do cuidado de enfermagem. João Pessoa, PB, Brasil, 2016

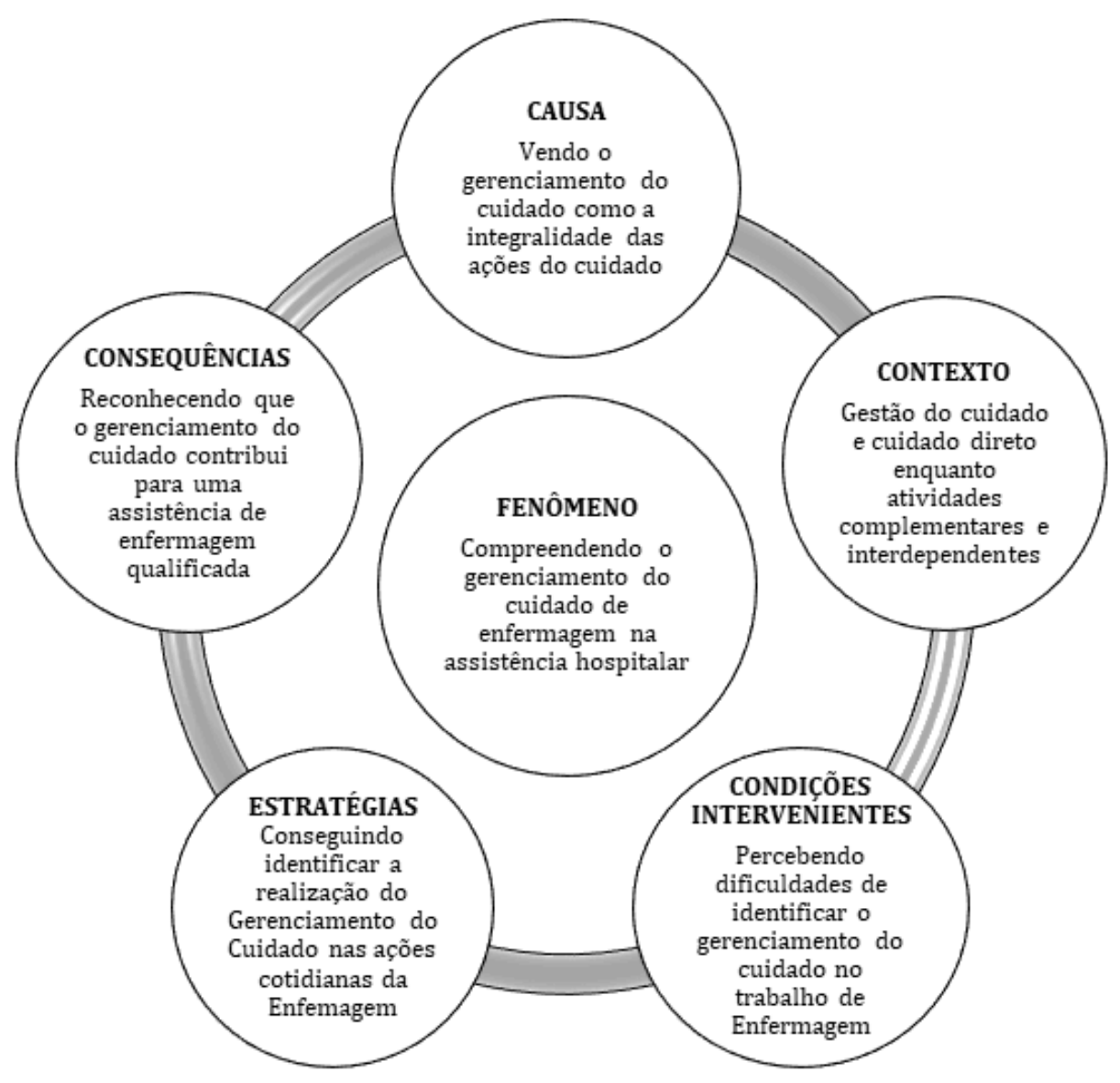

Fonte: dados da pesquisa, 2016

Este fenômeno descreve a aproximação dos enfermeiros com o tema "Gerenciamento do cuidado" e o modo como eles o vivenciam na sua prática. Os relatos demonstraram que, embora reconheçam a complementaridade das ações de gerência e cuidado e descrevam a importância do bom gerenciamento para a qualidade da assistência, estes profissionais não relacionam facilmente 0 gerenciamento do cuidado a sua prática, através dessa nomenclatura. Pode-se inferir que os mesmos não dão o devido reconhecimento à prática realizada e que se trata de um tema pouco discutido no cotidiano da enfermagem, requerendo empenho para transformar a realidade encontrada.

Destacando as condições causais do fenômeno, a percepção dos participantes do estudo sobre o gerenciamento do cuidado de Enfermagem resultou na categoria "Vendo o gerenciamento do cuidado como a integralidade das ações de cuidado", que resume todos os discursos dos profissionais, assim como, o significado da interface gerência e cuidado na prática profissional da Enfermagem, porquanto reúne as etapas e ações que compõem o processo de assistência ao paciente.

Assim, os enfermeiros descreveram o gerenciamento do cuidado de enfermagem como o conjunto de todas as atividades desenvolvidas no cotidiano do seu trabalho. Esse é um universo de ações diverso e complexo, porque abrange todo o processo do cuidado, composto por levantamento de problemas, construção de um plano de cuidados, implementação de ações, diretas e indiretas, com os pacientes e a avaliação contínua de cada uma dessas etapas, seus efeitos e a realização de ajustes durante todo esse percurso, conforme demonstra a fala a seguir:

[...] Significa o planejamento e organização de todas as ações voltadas ao cuidado do paciente. Incluindo desde a sistematização da assistência, ao abastecimento do setor, dimensionamento dos técnicos, ou seja, tudo que direta ou indiretamente influencia na qualidade da assistência prestada (Enf. 12). 
0 contexto envolvido no modo como os enfermeiros compreendem o gerenciamento do cuidado foi descrito através da categoria "Gestão do cuidado e cuidado direto vistos como atividades complementares e interdependentes", a qual demonstra a compreensão da indissociabilidade existente entre os conceitos e da contribuição do adequado gerenciamento para a realização de um cuidado de Enfermagem de boa qualidade:

[...] Para mim o gerenciamento é uma coisa imprescindível para que o cuidado de enfermagem se desenvolva. Você presta o cuidado e ao mesmo tempo supervisiona o trabalho da equipe, o funcionamento do setor, o estado do paciente, é uma coisa ligada a outra. Eu acho que um depende do outro, você faz as duas coisas ao mesmo tempo (Enf.5).

Enquanto condição interveniente foi constatada uma grande dificuldade inicial dos profissionais em reconhecer o gerenciamento do cuidado em sua prática de trabalho, conformando a categoria "Percebendo dificuldades em identificar o gerenciamento do cuidado no trabalho da Enfermagem". Essa dificuldade se apresentou no momento em que o entrevistador expôs o assunto da pesquisa e apresentou inicialmente o tema. Pouco a pouco foi sendo quebrada, através do desenvolvimento do discurso e do direcionamento da entrevista.

De tal modo, apesar da afirmação de que se tratava do relato da prática cotidiana e de atividades que compõem o dia a dia do trabalho dos profissionais, houve inicialmente uma reação de aversão à temática e às questões apresentadas, como mostra o fragmento que segue:

\section{[...] O que é gerenciamento do cuidado? Eu não sei responder isso não (Enf. 1).}

As estratégias de ação e interação apresentadas pelos enfermeiros para a prática do gerenciamento do cuidado de Enfermagem geraram a categoria "Conseguindo identificar a realização do gerenciamento do cuidado nas ações cotidianas da Enfermagem", que foi composta pelas subcategorias: "Realizando o gerenciamento do cuidado, através do planejamento da assistência"; "Gerenciando o cuidado, através da previsão de recursos materiais e humanos"; "Vendo o gerenciamento do cuidado como a realização de cuidados diretos e indiretos" e "Gerenciando o cuidado, através da distribuição de atribuições e do direcionamento do cuidado".

Todos esses aspectos discutidos nas subcategorias são ferramentas constituintes do gerenciamento do cuidado e que representam sua importância para a assistência de Enfermagem. Eles foram descritos, muitas vezes, de forma despretensiosa, nem sempre dentro do roteiro semiestruturado idealizado, o que demonstra que os rumos da pesquisa qualitativa são incertos e surpreendentes. Assim, apesar da resistência inicial, as falas dos participantes foram trazendo pontos muito importantes do gerenciamento do cuidado, que demonstram que essas conexões entre dimensões e atividades é algo mais presente do que imaginavam.

Dentro da subcategoria "Realizando o gerenciamento do cuidado através do planejamento da assistência", observa-se que o planejamento da assistência, evidenciado pela Sistematização da Assistência de Enfermagem (SAE), esteve presente repetidas vezes nos discursos dos enfermeiros e é visto como uma ferramenta para a realização do gerenciamento do cuidado e um dos pontos primordiais para o bom desenvolvimento da prática gerencial e assistencial. 0 que se pode observar nesta fala:

[...] O gerenciamento do cuidado de enfermagem significa um ordenamento do cuidado. Esse ordenamento é evidenciado pela SAE e por outros programas que contemplem o conhecimento teórico e a aplicação do mesmo na prática (Enf. 8).

A subcategoria "Gerenciando o cuidado através dos recursos materiais e humanos" revela que a previsão e a provisão de materiais também foram atribuições da Enfermagem relacionadas ao gerenciamento e ao bom andamento da assistência. Paralelamente a isso, o gerenciamento quantitativo e qualitativo dos recursos humanos também se mostrou bastante necessário.

Sabe-se que, o cuidado de enfermagem é permeado por ações de cuidados diretos, realizados em interação com o paciente e ações de cuidados indiretos, que ocorrem distante do paciente, porém visam seu benefício. A subcategoria "Vendo o gerenciamento de enfermagem como a implementação de cuidados" revela que elas se complementam, a fim de atingir o objetivo da assistência qualificada:

[...] Eu acho que seja a gente planejar a assistência ao paciente, isso de forma direta ou indireta, o que a gente tem que fazer (Enf. 1).

Dentro das estratégias de ação a subcategoria "Gerenciando o cuidado através da distribuição de atribuições e do direcionamento do cuidado" apresenta a descrição das atividades de gerenciamento 
do cuidado, como a distribuição das atividades e o direcionamento da equipe de Enfermagem para o cuidado. Esses elementos devem ser realizados pelo enfermeiro, com base no conhecimento técnicocientífico e com um planejamento adequado da assistência.

[...] Desde distribuir atribuições aos demais componentes da equipe, previsão de materiais e insumos que vão ser necessários, tudo isso fica a cargo da enfermagem (Enf. 10).

E por fim, diante desses elementos, identificou-se como consequências do fenômeno a categoria: "Reconhecendo que o gerenciamento do cuidado contribui para uma assistência de Enfermagem qualificada", em que se discutiu sobre o olhar dos enfermeiros de que o gerenciamento bem estabelecido qualifica o cuidado realizado. Considerando isso, os relatos dos profissionais demonstram a preocupação em oferecer a melhor assistência possível aos pacientes:

[...] Gerenciar o cuidado significa uma pessoa, que direciona os profissionais de enfermagem, para que, juntos consigam um fim que seja a boa assistência [...] Não é olhando para tá procurando defeito, mas procurando um caminho, porque gerenciar não é procurar defeito, gerenciar é procurar caminhos em que o cuidado seja melhor (Enf. 4).

Assim, a busca pela qualidade deve ser um compromisso diário dos profissionais de enfermagem, visto que, a realização de um trabalho em que se lida diretamente com pessoas e, frequentemente, em momentos de exposição de suas fragilidades, implica grande responsabilidade, ressaltando as estratégias que auxiliam nessa busca.

\section{Discussão}

Frente aos achados do estudo, a literatura descreve o gerenciamento do cuidado como um processo que envolve recursos materiais e humanos, a fim de alcançar os objetivos organizacionais, englobando desde o planejamento até o controle das ações realizadas (Mendes et al., 2011). Para além dessa afirmação, pode-se dizer que os objetivos almejados pelo gerenciamento do cuidado não são apenas organizacionais, mas também profissionais e de cuidado integral, sendo assim uma competência do enfermeiro ligada diretamente à qualidade assistencial (Soares et al., 2016).

Há que se ressaltar que o foco deve ser sempre o usuário e a realização de um cuidado eficaz. Assim, gerenciar o cuidado de enfermagem significa tê-lo como alvo das ações e dos processos que envolvem a assistência, incluindo o cuidado direto e o indireto, como as ações de planejamento, realização e delegação de tarefas, provimento de recursos, capacitação da equipe e a atuação do enfermeiro em espaços de articulação e negociação nos ambientes de trabalho (Chaves et al., 2012; Galiza et al., 2016).

Com base no fenômeno apresentado, pode-se afirmar que, a visão dos enfermeiros acerca da ligação entre as demandas gerenciais e o cuidado direto ao paciente na prática da assistência evidencia que esses profissionais estão inseridos em um processo de trabalho, organizado em subprocessos interligados e complementares. Nesse contexto, a gerência se apresenta como importante elo de articulação das atividades, de integração do processo de trabalho em saúde e dos diferentes membros que compõem a equipe (Santos et al., 2013; Montezelli et al., 2011; Dias et al., 2021).

Logo, gerenciar e cuidar não possuem sentidos dicotômicos, e sim complementares. Para que se possa alcançar o objetivo de oferecer um cuidado qualificado e resolutivo é necessário que haja a junção do saber-fazer gerenciar com o saber-fazer cuidar, de forma integrada e sistematizada (Christovam et al., 2012; Presotto et al., 2014). É essa relação que se busca encontrar nos ambientes de trabalho da Enfermagem, caso contrário, as ações serão cada vez mais compartimentalizadas e a integralidade da assistência, tão valiosa nesse processo, será perdida de vista.

As condições intervenientes do estudo revelaram que os enfermeiros têm dificuldades para conceituar as ações de gerência do cuidado em Enfermagem, pois muitos deles não compreendem ou desconhecem as múltiplas facetas constituintes do seu processo de trabalho. Essa realidade contribui ainda mais para que o trabalho da Enfermagem seja desvalorizado, tendo em vista serem aspectos que devem partir dos próprios profissionais (Christovam et al., 2012).

Observa-se que, muitas vezes a prática é desenvolvida de modo quase automático. Quando se anunciava que se tratava de uma pesquisa sobre gerenciamento do cuidado, a primeira reação era quase sempre negativa, de rejeição e de dificuldade para falar sobre um tema que é o resumo de todas as ações de enfermagem no desenvolvimento da assistência, como eles mesmos conseguiram perceber e descrever posteriormente. Por essa razão, é preciso pensar sobre as atividades que compõem o trabalho da Enfermagem e realizá-las de forma consciente, valorizando e tendo ciência de sua importância para o resultado final do cuidado prestado. 
Em consonância com as estratégias de ação e interação ligadas a prática do gerenciamento do cuidado de Enfermagem, a literatura evidencia que a SAE é o principal instrumento utilizado para o aperfeiçoamento e a qualificação do cuidado, conforme afirma pesquisa realizada na Região Sudeste do Brasil, uma vez que exige do enfermeiro conhecimento teórico-prático para melhorar a conduta, no que tange aos procedimentos de Enfermagem (Aguiar et al., 2010).

A implementação da SAE resulta ainda em uma forma melhor de organizar e efetivar o direcionamento das ações de enfermagem, com a otimização do tempo. Para tanto, são essenciais o comprometimento e o envolvimento de todos os profissionais com o trabalho, realizando a assistência sistematizada como parte integrante de um bom planejamento das ações (Mendes et al., 2011).

Para gerenciar o cuidado efetivamente, o enfermeiro deve planejar a assistência em sua unidade de trabalho. Essa é uma ação contínua, que reflete na realização de uma assistência qualificada e melhora a utilização tanto dos recursos financeiros quanto dos humanos, além de contribuir com o relacionamento entre gestores, usuários e organização (Santos et al., 2013). Esse planejamento se concretiza por meio da SAE, que requer que o enfermeiro norteie a equipe de Enfermagem e conheça a individualidade de cada paciente, a fim de oferecer um cuidado holístico e direcionado (Chaves et al., 2012).

Nesse contexto, entende-se que, quando o enfermeiro capacita sua equipe, planeja e delega ações, prevê e provê recursos humanos e materiais necessários, articula e negocia com a equipe multiprofissional, ele administra as tecnologias gerenciais a favor do cuidado, qualificando a assistência prestada (Santos \& Lima, 2011). A gestão do cuidado está diretamente relacionada à qualidade dos serviços de saúde e perpassa pelo uso adequado de todos os recursos materiais, pessoais e tecnológicos disponíveis e necessários à coordenação e à implementação das ações, sem perder de vista os valores morais e profissionais (Sol \& Farrez, 2010; Oliveira et al., 2012; Fernandes et al., 2016).

A pesquisa também discute sobre o dimensionamento do pessoal de Enfermagem, visto como uma importante ação de gerência do cuidado, porque se relaciona ao gerenciamento de recursos humanos e é responsável por abarcar a avaliação, a programação e a distribuição do quantitativo de pessoal necessário, bem como a análise de sua adequação às necessidades de cuidados de pacientes e familiares (Mendes et al., 2011).

Nortear a equipe de Enfermagem durante o cuidado também foi um ponto destacado nas estratégias de ação e interação. Corroborando com esse ponto, estudos trazem como algumas das finalidades do processo gerencial, o direcionamento do processo de trabalho, a garantia do bom funcionamento do serviço e das condições para se assistir com qualidade e a busca pelo equilíbrio nas relações (Manenti et al., 2012; Dias et al., 2021).

Isto porque, ao estar numa posição hierárquica de liderança, o enfermeiro tem a função de dirigir a equipe de Enfermagem e, em consequência desse papel, delega atividades aos trabalhadores sob sua supervisão. Delegar, nesse caso, significa fazer com que o trabalho seja repartido, mas que todos busquem atingir metas comuns. Na realidade, essa é uma necessidade do serviço e da atividade gerencial (Marquis \& Huston, 2015).

A gerência do cuidado de enfermagem no cenário hospitalar é descrita como uma prática sujeita a uma relação dialética entre o saber-fazer cuidar e o saber-fazer gerenciar, baseada em ações múltiplas e integradas, diretas e indiretas, com a finalidade de oferecer assistência integral e bem estruturada (Christovam et al., 2012; Fernandes et al., 2016). Ressalta-se que, mesmo as ações indiretas, têm a finalidade primeira de proporcionar bem-estar ao paciente, assim se houver a união dessas ações o cuidado prestado torna-se mais abrangente atendendo de forma mais eficaz as necessidades dos pacientes (Balbino \& Silvino, 2012; Dias et al., 2021).

No entanto, em acordo com os achados deste estudo, várias publicações mostram que os profissionais ainda têm dificuldades em reconhecer a importância das atividades gerenciais, consideradas como indiretas, vendo como relevantes apenas às atividades realizadas diretamente com o paciente, sem compreender a complementaridade dessas ações para a organização da assistência (Santos et al., 2013; Montezelli et al., 2011, Treviso et al., 2017). Por fim, destaca-se a necessidade de quebrar os paradigmas entre gerenciar e prestar o cuidado, a fim de que esse seja coeso, contínuo, qualificado e multidimensional.

\section{Considerações finais}

0 gerenciamento do cuidado de enfermagem é descrito como um conjunto de todas as atividades desenvolvidas pelo enfermeiro em sua prática, envolvendo o dimensionamento da equipe, planejamento, execução e avaliação das ações práticas assistenciais, prestadas direta ou indiretamente aos usuários dos serviços. 
Diante dessa constatação, os resultados deste estudo destacaram a importância da SAE e do conhecimento real das necessidades de cuidado e saúde dos pacientes, servindo de base para o planejamento da assistência e, consequentemente, para a prática de um gerenciamento do cuidado de qualidade. Contudo, faz-se necessário quebrar conceitos que separam as atividades gerenciais das assistenciais, porquanto ambas se articulam e complementam em busca de um cuidado qualificado e um trabalho profissional coeso e eficiente.

Evidencia-se que o uso da TFD, como abordagem metodológica deste estudo, permitiu o aprofundamento nas questões propostas, enriquecendo as discussões de cada ponto apresentado pelos profissionais, sob os aspectos organizacionais, individuais e o contexto político-social que os envolve.

Com isso, o estudo aponta a relevância do papel de gerente do cuidado desempenhado pelo enfermeiro e os desafios vivenciados em sua prática. Espera-se que os resultados desse estudo fomentem reflexões, bem como intervenções no processo de trabalho dos profissionais de enfermagem, no que concerne o desenvolvimento de novas competências para prestação de um cuidado integral, quebrando os paradigmas da dissociação de gerir e cuidar.

Nesse sentido, infere-se a necessidade de fortalecimento da interdisciplinaridade e estímulo maior para os âmbitos de ensino e pesquisa em gestão da saúde em Enfermagem, a fim de que os enfermeiros possam sair da graduação com uma base mais sólida de conhecimentos, aliada às ferramentas necessárias ao seu desempenho.

E ainda, ampliar os espaços de discussão sobre as experiências positivas e as negativas vivenciadas por esses profissionais no âmbito da alta gestão hospitalar, pois certamente, o relato das experiências são essenciais para a construção e implementação de estratégias viáveis para intervenção na prática, a fim de que os enfermeiros possam melhorar continuamente seu desempenho.

Apesar da limitação de ter sido realizado em apenas uma instituição pública, traz, enquanto contribuição para a enfermagem, reflexões acerca da necessidade de ampliar as discussões nessa temática, bem como, estimular a realização de novas pesquisas em diferentes contextos, a exemplo de instituições particulares, a fim de comparar e compreender como se dá o processo em outras realidades.

\section{Agradecimentos}

Esta pesquisa recebeu apoio financeiro da Coordenação de Aperfeiçoamento de Pessoal de Nível Superior (CAPES).

\section{Referências}

Aguiar, D. F. F., Stipp, M. A. C., Leite, L. J., Mattos, V. Z., \& Andrade, K. B. S. (2010). Gerenciamento de enfermagem: situações que facilitam ou dificultam o cuidado na unidade coronariana. Aquichán, 10(2), 115-131. http://www.scielo.org.co/pdf/aqui/v10n2/v10n2a03.pdf

Alves, C. A., Deslandes, S. F., \& Mitre, R. M. A. (2011). The management of nursing work in a pediatric ward of medium and high complexity: a discussion about co-management and humanization. Interface - Comunicação, Saúde, Educação, 15(37), 351-361. https://doi.org/10.1590/ $\underline{\mathrm{S} 1414-32832011005000008}$

Amestoy, S. C., Cestari, M. E., Thofehrn, M. B., Milbrath, V. M., \& Porto, A. R. (2010). Enfermeiras refletindo sobre o seu processo de trabalho. Cogitare Enfermagem, 15(1),158-163. http://doi.org/ 10.5380/ce.v15i1.17188

Balbino, C. M., \& Silvino, Z. R. (2012). The management of nursing care in the school community: a case study. Online Brazilian Journal Nursing, 11(2), 555-558. https://doi.org/ 10.5935/1676-4285.2012S032

Bertoncini, J. H., Pires, D. E. P., \& Ramos, F. R. S. (2011). Dimensões do trabalho da enfermagem em múltiplos cenários institucionais. Tempus Actas de Saúde Coletiva, 5(1), 123-133. http://doi.org/ $10.18569 /$ tempus.v5i1.922

Brasil, Ministério da Saúde (1986). Lei $n^{\circ} 7498$ de 25 de Junho de 1986. Dispõe sobre a regulamentação do exercício da enfermagem e dá outras providências. Diário Oficial da República Federativa do Brasil. http://www.planalto.gov.br/ccivil 03/leis/l7498.htm

Brasil, Ministério da Saúde. (2012). Conselho Nacional de Saúde. Resolução no 466, de 12 de dezembro de 2012. Aprova as diretrizes e normas regulamentadoras de pesquisa envolvendo seres humanos. Diário Oficial da União. https://conselho.saude.gov.br/resolucoes/2012/Reso466.pdf

Brasil, Ministério da Saúde. (2013). Política Nacional de Humanização da Atenção e Gestão do SUS. Secretaria de Atenção à Saúde. https://bvsms.saude.gov.br/bvs/publicacoes/ politica nacional humanizacao pnh folheto.pdf 
Cavalcante, A. M. R. Z., Brunori, E. H. F. R., Lopes, C. T., Silva, A. B. V., \& Herdman, T. H. (2015). Nursing diagnoses and interventions for a child after cardiac surgery in an intensive care unit. Revista Brasileira de Enfermagem, 68(1), 155-160. https://doi.org/10.1590/0034-7167.2015680121i

Chagas, M. S., \& Abrahão, A. L. (2017). Care production in health team focused on living work: the existence of life on death territory. Interface - Comunicação, Saúde, Educação, 21(63), 857-867. https://doi.org/10.1590/1807-57622016.0262

Chaves, L. D. P., Laus, A. M., \& Camelo, S. H. (2012). Ações gerenciais e assistenciais do enfermeiro em unidade de terapia intensiva. Revista Eletrônica de Enfermagem, 14(3), 671-678. https://doi.org/ 10.5216/ree.v14i3.15724

Christovam, B. P., Porto, I. S., \& Oliveira, D. C. (2012). Nursing care management in hospital settings: the building of a construct. Revista Escola de Enfermagem da USP, 46(3), 734-741. https://doi.org/ $10.1590 / \mathrm{S} 0080-62342012000300028$

Conselho Federal de Enfermagem (2017). Perfil da Enfermagem no Brasil - FIOCRUZ/COFEN, Rio de Janeiro, Volume I, Brasil. http://biblioteca.cofen.gov.br/wp-content/uploads/2019/05/ relatoriofinal.pdf

Dantas, C. C., Leite, J. L., Lima, S. B. S., Stipp, M. A. C. (2009). Grounded theory - conceptual and operational aspects: a method possible to be applied in nursing research. Rev Latino-Am. Enfermagem, 17(4): 573-579. https://www.scielo.br/i/rlae/a/IgPLBk4WD8bFVGSjwQnkHqC/? lang=en

Dias, C. F. C., Rabelo, S. K., Lima, S., B., S., Santos, T. M., Hoffmann, D. R. (2021). Gerenciamento do cuidado de enfermagem no contexto hospitalar: relato de experiência. Brazilian Journal of Health Review, 4(2):980-986. https://www.brazilianjournals.com/index.php/BJHR/article/viewFile/ $\underline{26695 / 21148}$

Faria, H. X., \& Araujo, M. D. (2010). Uma perspectiva de análise sobre o processo de trabalho em saúde: produção do cuidado e produção de sujeitos. Saúde e Sociedade, 19(2), 429-439. https://doi.org/ $\underline{10.1590 / \mathrm{S} 0104-12902010000200018}$

Galiza, F. T., Bezerra, A. L. S., Oliveira, A. S. S., Felipe, G. F., Fernandes, M. C., \& Lima, M. A. (2016). Management of the nursing care in the family health strategy. Revista Enfermagem UFPE on line, 10(11), 4075-4081. https://doi.org/10.5205/1981-8963-v10i11a11493p4075-4081-2016

Howard, P. F., Liang, Z., Leggat, S., Karimi, L. (2018). Validation of a management competency assessment tool for health service managers. Journal Health Organization Management, 32(1). https://doi.org/10.1108/JHOM-08-2017-0223

Fernandes, M. C, Silva, F. M. P., Barros, L. S. F., Pinto, L. M. C., Bezerra, I. C. B., Martins, A. P. A. (2016). Cuidar e gerenciar: práticas dialéticas ou dicotômicas no âmbito hospitalar das enfermeiras gerentes. Revista de Enfermagem UFPE on line, 10(3):985-990. https://periodicos.ufpe.br/revistas/ revistaenfermagem/article/view/11049/12459

Furukawa, P. O., \& Cunha, I. C. K. O. (2011). Profile and competencies of nurse managers at accredited hospitals. Revista Latino-Americana de Enfermagem, 9(1), 106-114. https://doi.org/10.1590/ $\underline{\text { S0104-11692011000100015 }}$

Garlet, E. R., Trindade, L. L., Lima, M. A. D. S, \& Bonilha, A. L. L. (2006) A resignificação dos processos gerenciais nas práticas do cuidado em enfermagem. Online Brazilian Journal Nursing, 5(3). http:// www.objnursing.uff.br/index.php/nursing/article/view/613/144

Giordani, J. N., Bisogno, S. B. C., \& Silva, L. A. A. (2012) Perception of nurses regarding management activities for user assistance. Acta Paulista de Enfermagem, 25(4), 511-516. https://doi.org/10.1590/ $\underline{\text { S0103-21002012000400005 }}$

Haussmann, M., \& Peduzzi, M. (2009). Articulação entre as dimensões gerencial e assistencial do processo de trabalho do enfermeiro. Texto \& Contexto Enfermagem, 18(2), 258-265. https:// www.scielo.br/pdf/tce/v18n2/08

Kurcgant, P. (2016). Gerenciamento em Enfermagem (3 ed.). Guanabara Koogan.

Leal, L. A., Soares, M. I., Silva, B. R., Chaves, L. D. P., Camelo, S. H. H. (2018). Desafios para desenvolver competências no âmbito hospitalar. Revista Mineira de Enfermagem, 22(e-1099). http:// www.dx.doi.org/10.5935/1415-2762.20180042

Lewis-Pierre, L. J., Kovacich, J., \& Amankwaa, L. (2017). The Application of Grounded Theory: An Example from Nursing Workforce Research. The Qualitative Report, 22(5), 1269-1283. https:// nsuworks.nova.edu/tqr/vol22/iss5/7/

Manenti, S. A., Ciampone, M. H. T., Mira, V. L., Minami, L. F., \& Soares, J. M. S. (2012). 0 processo de construção do perfil de competências gerenciais para enfermeiros coordenadores de área hospitalar. Revista da Escola de Enfermagem da USP, 46(3), 727-733. https://doi.org/10.1590/ $\underline{\text { S0080-62342012000300027 }}$ 
Marquis, B. L., Huston, C. J. (2015). Administração e liderança em enfermagem: teoria e aplicação (8 ed.). Artes Médicas.

Massaro, M., \& Chaves, L. D. P. (2009). A produção científica sobre gerenciamento em enfermagem hospitalar: uma pesquisa bibliográfica. Cogitare Enfermagem, 14(1), 150-158. http://doi.org/ $10.5380 /$ ce.v14i1.14135

Metelski, F. K., Alves, T. F., Rosa R., Santos, J. L. G., Andrade, S. R. (2020). Dimensões da gestão do cuidado na prática do enfermeiro na atenção primária: revisão integrativa. Revista de Enfermagem da UERJ, 28(e51457). https://doi.org/10.12957/reuerj.2020.51457

Mendes, R. F., Gama, B. M. B. M., Brito, M. J. M., \& Vianna, M. S. (2011). Significados e possibilidades que tecem a gerência em enfermagem - o compromisso com a assistência. Revista de Enfermagem do Centro Oeste Mineiro, 1(2), 176-189. https://doi.org/10.19175/recom.v0i0.67

Merhy, E. E., Chakkour, M. (1997). Em busca de ferramentas analisadoras das tecnologias em saúde: a informação e o dia a dia de um serviço, interrogando e gerindo o trabalho em saúde. In: E. E. Merhy, \& R. Onocko. Agir em saúde: um desafio para o público (2 ed., pp. 113-160). Hucitec.

Montezelli, J. H., Peres, A. M., \& Bernardino, E. (2011). Demandas institucionais e demandas do cuidado no gerenciamento de enfermeiros em um pronto socorro. Revista Brasileira de Enfermagem, 64(2), 348-354. https://doi.org/10.1590/S0034-71672011000200020

Montezeli, J. H.; Caveião, C.; Hey, A. P.; Peres, A. M.; Romanio, M. S.; Tavares, Z. F. (2016). Leadership mobilization: conception of undergraduate nursing students. Journal of Research: Fundamental Care Online, 8(4), 5114-5119. https://doi.org/10.9789/2175-5361.2016.v8i4.5114-5119

Morais, F. R. C., Silva, C. M. C., Ribeiro, M. C. M., Pinto, N. R. S., \& Santos, I. (2011). Resgatando o cuidado de enfermagem como prática de manutenção da vida: concepções de Collière. Revista Enfermagem UERJ, 19(2), 305-310. http://www.facenf.uerj.br/v19n2/v19n2a22.pdf

Oliveira, F. E. L., Fernandes, S. C. A., Oliveira, L. L., Queiroz, J. C., \& Azevedo, V. R. C. (2012). A gerência do enfermeiro na estratégia saúde da família. Revista da Rede de Enfermagem do Nordeste, 13(4), 834-844. http://www.periodicos.ufc.br/rene/article/view/4043/3171

Pires, D. (2009). A enfermagem enquanto disciplina, profissão e trabalho. Revista Brasileira de Enfermagem, 62(5), 739-44. https://doi.org/10.1590/S0034-71672009000500015

Presotto, G. V., Ferreira, M. B. G., Contim, D., \& Simões, A. L. A. (2014). Dimensões do trabalho do enfermeiro no contexto hospitalar. Revista da Rede de Enfermagem do Nordeste, 15(5), 760-770. https://doi.org/10.15253/2175-6783.2014000500005

Santos, M. C., \& Bernardes, A. (2010). Comunicação da equipe de enfermagem e a relação com a gerência nas instituições de saúde. Revista Gaúcha de Enfermagem, 31(2), 359-366. https://doi.org/ $10.1590 /$ S1983-14472010000200022

Santos, J. L. G., \& Lima, M. A. D. S. (2011). Gerenciamento do cuidado: ações dos enfermeiros em um serviço hospitalar de emergência. Revista Gaúcha de Enfermagem, 32(4), 695-702. http://doi.org/ $\underline{10.1590 / \text { S1983-14472011000400009 }}$

Santos, J. L. G., Prochnow, A. G., Lima, S. B. S., Leite, J. L., \& Erdmann, A. L. (2011). Communication conceptions in Hospital Nursing Management between head nurses in a University Hospital. Revista Escola de Enfermagem da USP, 45(4), 959-965. https://doi.org/10.1590/ $\underline{\text { S0080-62342011000400024 }}$

Santos, J. L. G., Prochnow, A. G., Silva, D. C., Cassettari, S. S. R., Guerra, S. T., Erdmann, A. L. (2012). Comunicação gerencial na enfermagem hospitalar: dificuldades e estratégias para superá-las - estudo descritivo. Online Brazilian Journal Nursing, 11(2). https://doi.org/10.5935/1676-4285.20120034

Santos, J. L. G., Pestana, A. L., Guerrero, P., Meirelles, B. S. H., \& Erdmann, A. L. (2013). Práticas de enfermeiros na gerência do cuidado em enfermagem e saúde: revisão integrativa. Revista Brasileira de Enfermagem, 66(2), 257-263. https://doi.org/10.1590/S0034-71672013000200016

Soares, M. I., Camelo, S. H. H., Resck, Z. M. R., Terra, F. S. (2016). Nurses' managerial knowledge in the hospital setting. Revista Brasileira de Enfermagem, 69(4). http://dx.doi.org/ 10.1590/0034-7167.2016690409i

Soder, R. M., Santos, L. E., Oliveira, I. C., Silva, L. A. A., Peiter, C. C., Santos, J. L. G. (2020) Healthcare management practices in primary care. Revista Cubana de Enfermagem, 36(1):e2815. http:// www.revenfermeria.sld.cu/index.php/enf/article/view/2815

Sol, A. A., \& Farrez, R. (2010). Influencia de la gestión del cuidado en la calidad de la atención en salud. Revista Cubana de Enfermería, 26(2), 14-26. http://scielo.sld.cu/pdf/enf/v26n2/enf03210.pdf

Strauss, A., Corbin, J. (2008). Pesquisa Qualitativa: Técnica e procedimentos para o desenvolvimento da teoria fundamentada (2 ed). Artmed.

Treviso, P., Peres, S. C., Silva, A. D., \& Santos, A. A. (2017). Competências do enfermeiro na gestão do cuidado. Revista de Administração em Saúde, 17(69), 1-15. http://doi.org/10.23973/ras.69.59 